\title{
Mobility Assessment of the Supraspinatus in a Porcine Cadaver Model Using a Sensor-Enhanced, Arthroscopic Grasper
}

\author{
Felix Porschke, ${ }^{1}$ Christoph Luecke,,${ }^{1}$ Thorsten Guehring, ${ }^{2}$ \\ Christel Weiss, ${ }^{3}$ Stefan Studier-Fischer, ${ }^{1}$ Paul Alfred Gruetzner, ${ }^{1}$ \\ and MARC SChNetzKe ${ }^{1,4}$
}

\begin{abstract}
${ }^{1}$ BG Trauma Center Ludwigshafen at Heidelberg University Hospital, Ludwig-Guttmann-Straße 13, 67071 Ludwigshafen, Germany; ${ }^{2}$ Arcus Sportklinik, Rastatter Str. 17-19, 75179 Pforzheim, Germany; ${ }^{3}$ Department of Medical Statistics, University Medicine Mannheim, Medical Faculty Mannheim of the University of Heidelberg, 68167 Mannheim, Germany; and ${ }^{4}$ German Joint Center, Atos Clinic, Bismarckstraße 9-15, 69115 Heidelberg, Germany
\end{abstract}

(Received 18 December 2019; accepted 14 July 2020; published online 13 August 2020)

Associate Editor Michael R. Torry oversaw the review of this article.

\begin{abstract}
Tendon mobility is highly relevant in rotator cuff surgery. Objective data about rotator cuff mobility is rare. Tendon mobility still needs to be evaluated subjectively by the surgeon. This study aims to establish a porcine animal model for mobility analysis of the supraspinatus. In this context, we introduce a sensor-enhanced, arthroscopic grasper (SEAG) suitable for objective intraoperative measurements of tendon mobility in clinical praxis. Tendon mobility of 15 fresh porcine cadaver shoulders with artificial rotator cuff tears was evaluated using the SEAG. Mobility characteristics (load-displacement curves, maximum load, stiffness) were studied and inter- and intraobserver agreement (intraclass correlation coefficient (ICC)) were tested. Factors with a potential adverse effect (plastic deformation and rigor mortis) were also evaluated. All shoulders showed characteristic reproducible load-displacement curves with a nonlinear part at the start, followed by a linear part. Mean maximum load was $28.6 \mathrm{~N} \pm 12.5$. Mean stiffness was $6.0 \mathrm{~N} / \mathrm{mm} \pm 2.6$. We found substantial interobserver agreement (ICC 0.672) and nearly perfect intraobserver agreement (0.944) for maximum load measurement. Inter- $(0.021)$ and intraobserver (0.774) agreement for stiffness was lower. Plastic deformation and rigor mortis were excluded. The animal model demonstrates reliable and in vivo-like measurements of tendon mobility. The SEAG is a reliable tool for tendon mobility assessment.
\end{abstract}

Keywords-Tendon, Rotator cuff, Arthroscopy, Biomechanics.

Address correspondence to Marc Schnetzke, German Joint Center, Atos Clinic, Bismarckstraße 9-15, 69115 Heidelberg, Germany. Electronic mail: marc.schnetzke@atos.de

\section{ABBREVIATIONS}

SEAG Sensor-enhanced, arthroscopic grasper ICC Intraclass correlation

\section{INTRODUCTION}

Rotator cuff injury is a frequent musculoskeletal disease and causes pain and functional impairment. Reconstruction of rotator cuff defects has become increasingly important due to advances in surgical techniques and the development of new implants. Despite such progress, a considerable percentage of patients still suffers from a re-tear after tendon reconstruction. ${ }^{15,34}$ Clinical and experimental research has focused on the insertion site (bone-implant and implant-tendon interface) of tendon reconstruction. The biomechanical properties of various repair techniques have been thoroughly investigated using animal or human cadaver models.

Due to the natural course of tendon retraction and remodeling, the intraoperative mobility of a torn tendon is also an important factor. ${ }^{14,28}$ Several radiological parameters have been identified as predictors for clinical outcome and re-tear rates. ${ }^{26,33}$ Tendon mobility could be an important link or even an explanation for worse outcomes in the presence of a retracted tendon $^{17,28}$ or atrophy of the muscle ${ }^{14,37}$ in MRI. 
In addition, tendon mobility is an important factor to consider in decisions about the best individual surgical procedure. Different surgical methods have been implemented to improve mobility and therefore reduce the strain of torn tendons in rotator cuff reconstruction. . $^{1,18,25}$ Tauro et al. introduced the arthroscopic interval slide procedure and reported favorable results in comparison to non-released shoulders. ${ }^{36}$ These results were reproduced by Lo and Burkhardt. They even advanced this procedure with additional posterior release. ${ }^{25}$

Recently, worse outcomes for patients with high tendon tension at time of reconstruction were found. ${ }^{16,31}$ Mobility might therefore prescribe the reconstruction technique. ${ }^{5,19}$

Despite the growing interest in tendon mobility, objective measurement methods for it are still lacking. Therefore, surgeons need to apply their experience to assess the tendon mobility correctly. Furthermore, no model exists for investigating rotator cuff tendon mobility. Several in vitro studies have focused on tensile properties of exemplary parts of the rotator cuff. ${ }^{10,12,21}$ However, the entire myotendinous unit has rarely been investigated.

The aim of this feasibility study was to establish an in vivo-like model for mobility analyses of the supraspinatus myotendinous unit. A second aim was to introduce a prototype of a sensor-enhanced, arthroscopic grasper (SEAG) for objective tendon mobility measurements which could be transferred to the clinical setting. The following design criteria were set to ensure practicability in clinical praxis: The SEAG must be portable, have similar dimensions to standard arthroscopic instruments, and be sterilizable. Further grasping and the release of the tendon stump should be fast and intuitive to avoid unnecessary extension of the operation. With regard to existing data, the SEAG must provide high accuracy and a measuring range between 0.5 and $100 \mathrm{~N}^{4}$ This was achieved by connecting a commercial reusable arthroscopic grasper with self-releasing locking mechanism via a sterilizable custom-made aluminum fast adapter (Fa. Surgitaix) to an industrial force gauge. Furthermore, tendon mobility measurement using the SEAG must fulfill the criteria of reliability. We therefore analyzed forcedisplacement characteristics of 15 porcine supraspinatus tendons measured by the SEAG and evaluated inter- and intraobserver agreement.

\section{MATERIALS AND METHODS}

\section{Specimen Preparation}

15 porcine shoulders were harvested from 15 euthanized domestic pigs (Sus scrofa forma domestica). Due to their original purpose for the food industry no approval of an animal welfare organization was needed. Within 50 min postmortem, the shoulders were transferred to the laboratory and prepared. After cyclic preloading $(10 \mathrm{~min})$ the first measurements started $1 \mathrm{~h}$ postmortem. Harvesting, preparation, and all measurements were completed within $2 \mathrm{~h}$ postmortem using a rigorous schedule to guarantee in vivo-like tissue properties ${ }^{38}$ (Fig. 1).

The laboratory air-conditioning system kept the ambient temperature constant at $20{ }^{\circ} \mathrm{C} \pm 0.5$ and humidity at $45 \% \pm 2.5$.

Each shoulder was disarticulated from the thorax, by avoiding any damage to the subscapularis, supraspinatus, infraspinatus, and deltoid. The humerus was exarticulated in the elbow joint. Biceps and coracobrachialis muscles were resected distally at the height of the elbow joint.

The scapula was fixed using three bicortical screws (superior angle, inferior angle and glenoid neck) with the medial border perpendicular to the ground and the subscapularis facing a mounting plate to the testing station. Contact pressure between the mounting plate and subscapularis muscle was prevented by screw nuts used as spacers between the shoulders and the testing station. The humeral shaft was distally fixed at $30^{\circ}$ glenohumeral abduction using another bicortical screw.

Skin and subcutaneous fat were removed. The deltoid muscle was detached from its insertion at the proximal humerus. The distal portion of the muscle was resected allowing access to the supraspinatus insertion, while the proximal part of the muscle was not touched to preserve the anatomic sliding layers.

\section{Defect Creation}

The supraspinatus tendon was detached from its insertion site on the proximal humerus to mimic a fullthickness tear without retraction. The tendon width (distance between anterior and posterior tendon margin at the lateral tendon stump) and length of the entire myotendinous unit (from most medial insertion of supraspinatus muscle to lateral tendon stump) were obtained using a digital caliper.

\section{Testing Station}

The custom-designed testing station was built from $23.5 \mathrm{~mm}$ aluminum rectangular tubes and perforated plates (Fig. 2a). A unique feature was a $317 \mathrm{~mm} \times 157 \mathrm{~mm}$ slide for the SEAG, which enables lateralization of the SEAG in millimeter intervals using an isometric screw (M6 acc. DIN 13-1). Additional 


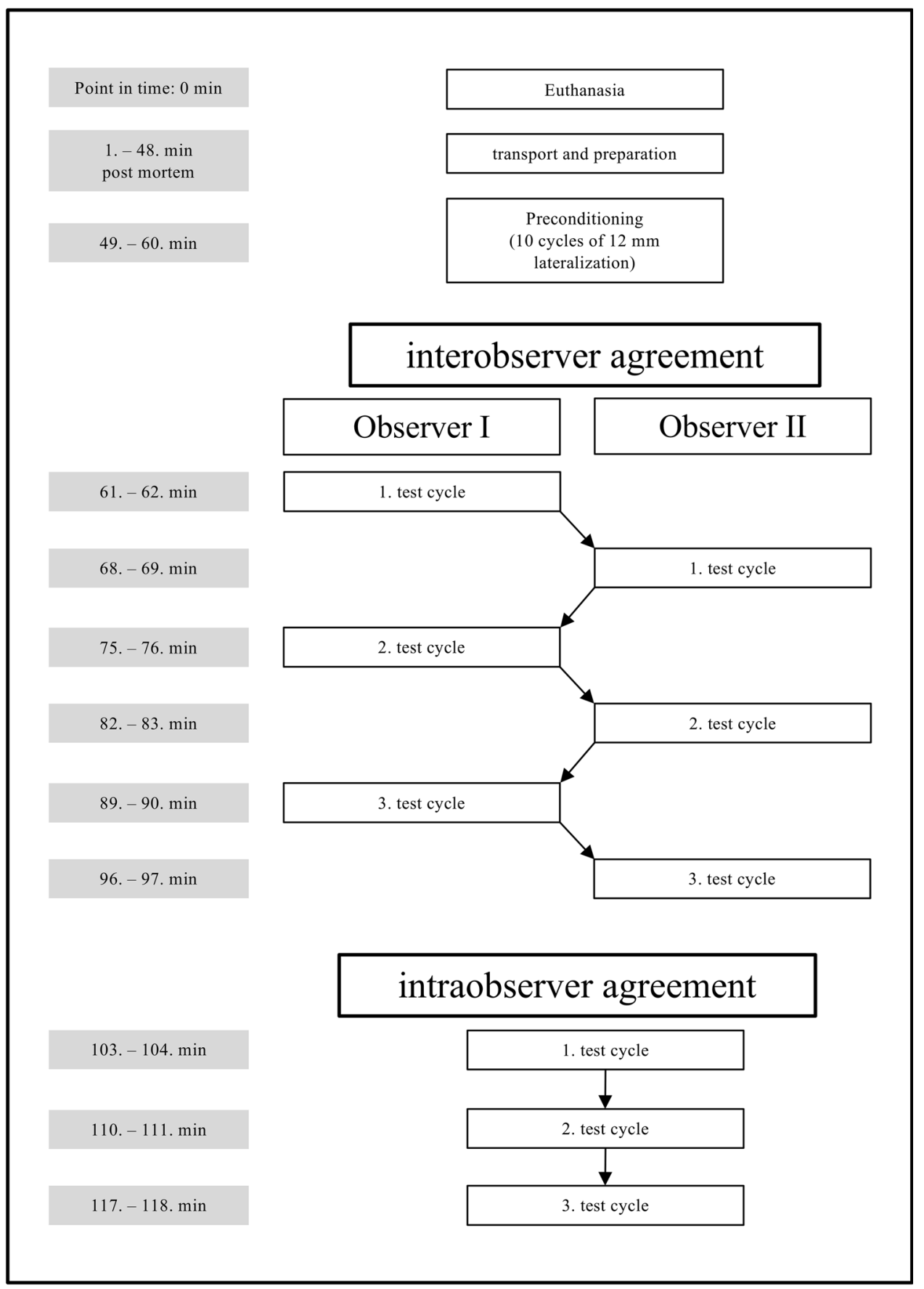

FIGURE 1. Test protocol with timetable.

cross braces were used to prevent distortion of any part of the testing station.

\section{Sensor Enhanced Arthroscopic Grasper}

For the SEAG, a commercial arthroscopic grasper (Rotator cuff grasper: $4.2 \mathrm{~mm}$ shaft diameter with traumatic jaws, and a self-releasing locking mechanism from Fa. Arthrex Inc.) was connected in series via a custom-made aluminum fast adapter (Fa. Surgitaix) to an industrial force gauge (PCE-FB 200, accuracy $\pm 0.1 \%$, precision $0.05 \mathrm{~N}$, measuring range 0.5 200 N, Fa. PCE Instruments) (Fig. 2b). The arthroscopic grasper and adapter are eligible for autoclave sterilization.

If a standard sterile drape for the force gauge is also used, the SEAG can be used in sterile surgical procedures. A Bluetooth interface facilitates wireless in time 

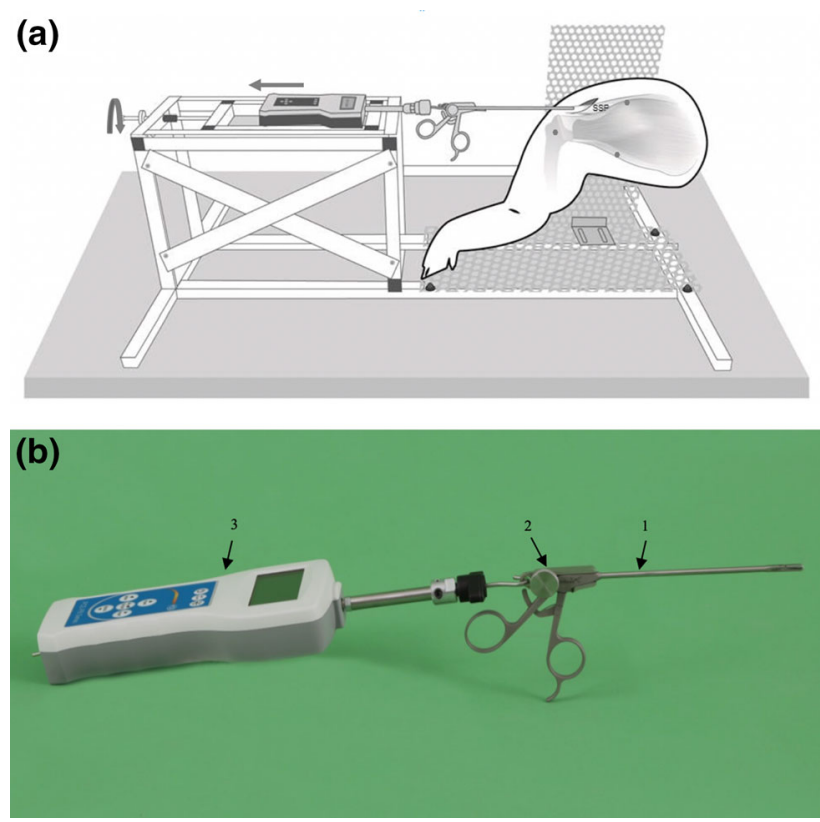

FIGURE 2. (a) Schematic illustration of the custom-designed testing station. The SEAG is aligned with the supraspinatus muscle (SSP) and grasps the distal tendon stump. The SEAG is lateralized in $1 \mathrm{~mm}$ steps via a slide by using an isometric screw (left). (b) Sensor enhanced arthroscopic grasper (SEAG): An arthroscopic grasper (1) is connected in series via a custom-made aluminum fast adapter (2) to a force gauge (3).

transfer of the measured data to a personal computer for further data processing and storage.

The SEAG was attached to the testing station and was aligned with the tendon and muscle fibers of the supraspinatus.

\section{Measurement of Mobility}

After grasping the distal tendon stump, the slide and hence the SEAG was lateralized stepwise, and the tendon stump was therefore displaced. Since the SEAG was aligned with the tendon and muscle fibers, longitudinal force was applied.

The digital force gauge allowed real-time measurement of the force loaded on the supraspinatus in Newton (Fig. 2a).

First, the tendon was preconditioned with ten cycles of $12 \mathrm{~mm}$ lateralization $(1 \mathrm{~mm} / 5 \mathrm{~s})$. The tendon was then preloaded with $0.1 \mathrm{~N}$ to define the starting point $(0 \mathrm{~mm})$.

The measurement was then performed by lateralizing the tendon in $1 \mathrm{~mm}$ steps to a maximum of $12 \mathrm{~mm}$. Preliminary testing had shown a risk of tendon slippage if a tendon lateralization higher than $12 \mathrm{~mm}$ was used. After each lateralization step $(1 \mathrm{~mm})$ and an equilibrium period of $5 \mathrm{~s}$, the load in Newton was registered by the tensiometer. A test cycle was finished at $12 \mathrm{~mm}$ lateralization.
Using this approach, the mobility of the tendon was drawn in a force-displacement graph $(\mathrm{N} / \mathrm{mm})$.

Two different parameters were defined to describe tendon mobility in this study: maximum load on the tendon within $12 \mathrm{~mm}$ of lateralization and stiffness of the tendon, computed as the slope in the linear region of the force-displacement curve $(10-12 \mathrm{~mm}) .{ }^{39}$ Both were tested for interobserver and intraobserver agreement.

Obvious degenerative changes of the glenohumeral joint (1), preexisting defect of the rotator cuff (2) and damage of rotator cuff before definite measurements started (3) lead to exclusion of the specimen. In the case of tendon slippage within intraobserver agreement measurements (4) and duration of measurements $>2 \mathrm{~h}$ postmortem (5) the shoulders were excluded as well. Data from these shoulders were not used.

\section{Testing Protocol}

\section{Interobserver Agreement}

Interobserver agreement testing was performed to evaluate the influence of attaching and detaching the grasper and the individual definition of the starting point. It is assumed that the actual measurement (after attachment of the grasper) as described above is very reliable because it is controlled mechanically by the test station. However, the moment of attaching the grasper and determining the starting point is vulnerable to ambiguity. Therefore two observers tested the interobserver agreement.

After preconditioning, the SEAG was detached from the tendon and the slide was brought in minimum lateralization (default position). Observer I then grasped the tendon and defined the starting point at $0.1 \mathrm{~N}$. Then the first test cycle was carried out by observer I as described in the section "measurement of mobility".

At the end of the test cycle, the SEAG was detached and the slide was brought to the default position. After grasping the tendon again and defining his own starting point at $0.1 \mathrm{~N}$, observer II carried out his first test cycle in the same manner. Overall, both observers performed three test cycles in turn. Interobserver agreement was tested for maximum load and stiffness was calculated (Fig. 1).

\section{Intraobserver Agreement}

Intraobserver agreement was performed to evaluate the actual measurements. Therefore the SEAG was not detached between the test cycles.

The starting point was once again defined after preload, and the first test cycle was performed. After reaching the endpoint of $12 \mathrm{~mm}$, the SEAG was not 
detached but brought back exactly $12 \mathrm{~mm}$ to the starting point. This was repeated three times without detaching the SEAG. Again agreement for maximum load and stiffness were calculated (Fig. 1).

\section{Force-Displacement Characteristics}

Due to the lack of an existing gold standard for comparative validation, we decided to evaluate the force-displacement characteristics and tested the correlation between lateralization and load. Therefore data from all nine test cycles was used. The findings were compared to the few existing values about in vivo tension of human rotator cuff for comparative purposes. Change of mobility over time was also examined to exclude plastic deformation or rigor mortis.

\section{Statistical Analysis}

Statistical analysis was performed by a biometrician from the Medical Faculty Mannheim of Heidelberg University, using the statistical software SAS, Release 9.4, (SAS Institute, Cary, NC, USA). Data have been entered in Microsoft ${ }^{\circledR}$ Excel for Mac Version 15.22 (Microsoft ${ }^{\circledR}$, Redmond, USA) and then imported into SAS.

For quantitative variables, mean and standard deviation were calculated. For the assessment of the inter- and intraobserver reliability, the intraclass correlation (ICC) was calculated for every lateralization step based on a 2-way ANOVA.

Agreement strength was inferred from the ICC in accordance with the recommendations of Landis and Koch. ${ }^{23}$ ICC $<0.20$ was interpreted as poor agreement, a value in the range of 0.21 to 0.40 as fair agreement, between 0.41 and 0.60 as moderate agreement, in the range 0.61 to 0.80 as substantial agreement, and above 0.81 as nearly perfect agreement.

The correlation between load and lateralization was calculated using Spearman's correlation coefficient. Deviation of mobility over time was evaluated using an ANOVA for repeated measurements with post hoc testing according to Tukey-Kramer using the SAS procedure PROC MIXED. In general, a test result was considered statistically significant if the corresponding $p$ value was less than 0.05 .

\section{RESULTS}

The sample consisted of 7 left and 8 right shoulders. All prepared shoulders showed an intact rotator cuff without degenerative joint changes. Mean width of the tendon stump was $11.2 \mathrm{~mm} \pm 2.3$. Mean length of the myotendinous unit was $44.5 \mathrm{~mm} \pm 3.5$, which resulted
TABLE 1. Tendon measurements and interclass correlation for inter- and intraobserver reliability.

\begin{tabular}{|c|c|c|}
\hline & & 15 shoulders \\
\hline \multicolumn{3}{|l|}{ Side } \\
\hline \multicolumn{2}{|l|}{ Right } & 8 \\
\hline \multicolumn{2}{|l|}{ Left } & 7 \\
\hline \multicolumn{2}{|c|}{ Myotendinous length in $\mathrm{mm}$} & $44.4 \pm 3.4$ \\
\hline \multicolumn{2}{|c|}{ Distal Tendon width in mm } & $11.4 \pm 2.3$ \\
\hline \multicolumn{2}{|c|}{ Rel. myotendinous lenghthening in \% } & $27 \pm 2$ \\
\hline & $\begin{array}{c}\text { Interobserver } \\
\text { agreement }\end{array}$ & $\begin{array}{c}\text { Intraobserver } \\
\text { agreement }\end{array}$ \\
\hline Maximal load (N) & $29.8 \pm 13.3$ & $26.6 \pm 11.6$ \\
\hline Stiffness (N/mm) & $5.1 \pm 2.1$ & $6.9 \pm 3.1$ \\
\hline Max. ICC & 0.672 & 0.944 \\
\hline
\end{tabular}

Categorical data as categorical data as frequencies and continuous data as mean \pm standard deviation.

in a mean relative lengthening of $27 \% \pm 2$ for maximal lateralization (Table 1). Tendon slippage was observed once and the shoulder concerned was excluded. For all included shoulders, the measurements were completed within $2 \mathrm{~h}$ postmortem.

The maximum load was found at $12 \mathrm{~mm}$ lateralization in all shoulders and test cycles and had a mean value of $28.6 \mathrm{~N} \pm 12.5$. The width of the tendon stump or length of the myotendinous unit did not affect the maximum load ( $p=0.608 ; p=0.972)$.

In all test cycles, the load increased with progressing lateralization. It started with a non-linear part followed by a linear part (Figs. 3a and 3b). The Spearman's correlation coefficient of 0.92 confirms the strong correlation between load and lateralization $(p<0.001)$. The mean calculated stiffness was $6.0 \mathrm{~N} /$ $\mathrm{mm} \pm 2.6$. There was no significant change over time for maximum load or calculated stiffness within $2 \mathrm{~h}$ postmortem $(p=0.427 ; p=0.704)$.

\section{Interobserver Agreement}

Interobserver agreement was strongly affected by the lateralization (Table 2). For load, the highest ICC values between both observers were found at maximum lateralization and demonstrated a substantial interobserver agreement (ICC 0.672). The interobserver agreement for calculated stiffness was 0.021 (slight agreement).

\section{Intraobserver Agreement}

For intraobserver agreement, a maximum ICC of 0.944 ("nearly perfect" agreement) was found (load). The reliability showed a strong association with the grade of lateralization (Table 2). Calculated stiffness showed an ICC of 0.774 (substantial agreement). 
(a)

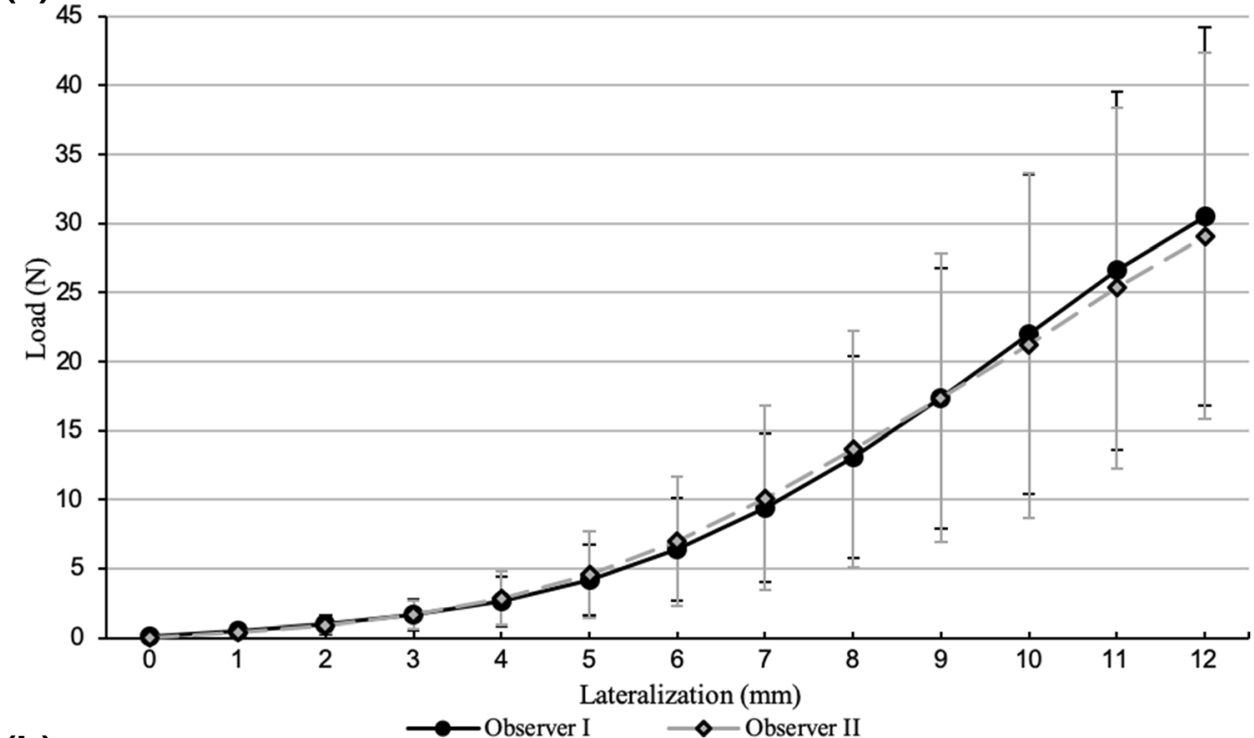

(b)

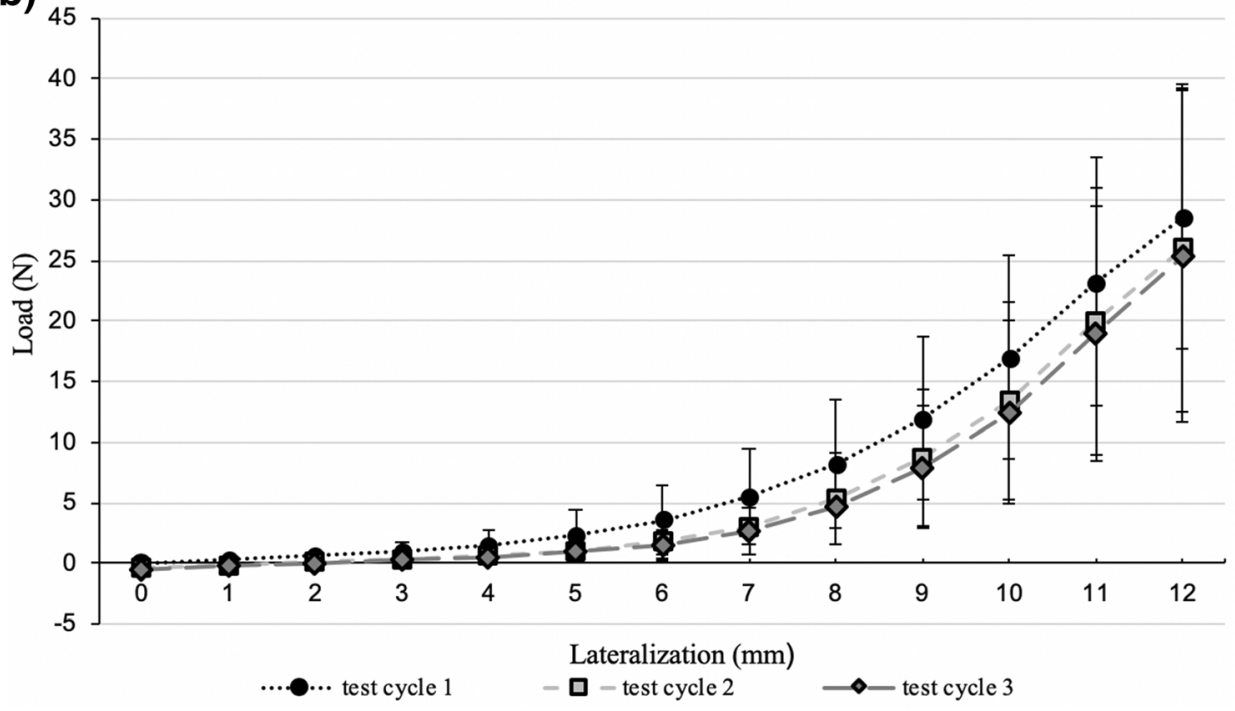

FIGURE 3. (a) Force-displacement graph for interobserver agreement measurements (in mean and \pm for all shoulders) observer I vs. observer II. (b) Force-displacement graph for intraobserver agreement measurements (in mean and \pm of all 15 shoulder) for 3 test cycles

\section{DISCUSSION}

The aim of this study was to implement an animal model that provides biomechanical properties close to in vivo tissue for exploring the mobility of the supraspinatus myotendinous unit. A second aim was to introduce the SEAG, a novel device for tension measurement of the rotator cuff.

Our measurements reveal reproducible load-displacement curves for all tested shoulders. All shoulders exhibit nonlinear curves at the start of lateralization (toe-in region) followed by a linear section. This characteristic load-displacement correlation was previously reported for in vitro and in vivo tendons. ${ }^{9,35}$
The mean maximum load on the myotendinous unit was $28.6 \mathrm{~N}$. This is quite similar to the intraoperative repair tension of in vivo human supraspinatus tendons $\left(31.9 \mathrm{~N}^{16} 28.5 \mathrm{~N}^{31}\right)$. The Spearman's correlation coefficient confirms the strong correlation between load and lateralization $(p<0.001)$.

We could demonstrate substantial interobserver agreement and nearly perfect intraobserver agreement for the assessment of maximum load. Conversely, ICCs for calculated stiffness were quite low. ICC for the intraobserver agreement was 0.774 and for interobserver agreement 0.021 . We found a mean calculated stiffness of $6.0 \mathrm{~N} / \mathrm{mm}$, which is much lower compared to existing data about stiffness of isolated tendons. ${ }^{7}$ 
TABLE 2. Results of objectivity and reliability testing (intraclass correlation) for all 15 shoulders.

\begin{tabular}{lcc}
\hline $\begin{array}{l}\text { Lateralization } \\
(\mathrm{mm})\end{array}$ & $\begin{array}{c}\text { Interobserver } \\
\text { agreement }\end{array}$ & $\begin{array}{c}\text { Intraobserver } \\
\text { agreement }\end{array}$ \\
\hline 1 & 0.316 & 0.206 \\
2 & 0.287 & 0.162 \\
3 & 0.325 & 0.159 \\
4 & 0.331 & 0.230 \\
5 & 0.414 & 0.363 \\
6 & 0.491 & 0.520 \\
7 & 0.578 & 0.594 \\
8 & 0.635 & 0.684 \\
9 & 0.622 & 0.771 \\
10 & 0.657 & 0.849 \\
11 & 0.672 & 0.914 \\
12 & 0.624 & 0.944 \\
\hline
\end{tabular}

Based on these findings, we cannot recommend using stiffness for mobility characterization.

Confounders such as plastic deformation of the tendon or effects of rigor mortis could be excluded.

In conclusion, we present a valid and reliable model for the clinically important but scientifically underrepresented topic of tendon mobility. We implemented a in vivo-close model by using fresh porcine shoulders within $2 \mathrm{~h}$ postmortem.

As it meets the design criteria of portability, sterilizability, and fast and intuitive handling in combination with high accuracy and reliable measurements of mobility, the SEAG is suitable for use in everyday clinical practice. Various shoulder models have been used in biomechanical studies. ${ }^{6}$ Porcine shoulders have been identified as a useful, cost-effective, and consistent model, and are widely used for research on rotator cuff surgery. Most of the studies investigated the insertion site after tendon reconstruction, especially bone-implant (type, number, and position of anchors) and implant-tendon interface (suture material, type, and number of knots). Parameters like load to failure or gap formation were the main focus. ${ }^{13,29}$ In these studies, the supraspinatus muscle was often dissected. Biomechanical properties, especially the mobility of the myotendinous unit, were neglected.

Some studies have elaborately engaged with the biomechanical properties of human rotator cuff tendon. Biomechanical, and often histological, parameters were analyzed using mostly small probes in fresh-frozen cadaver shoulders. ${ }^{10,12,22}$ This basic research accurately specified the biomechanical parameters of different parts of the tendon. However, this approach has limitations since the reaction of the entire muscletendon unit is far more complicated on loading.

For small animals, especially rats, biomechanical in vivo studies have tested the entire myotendinous complex. ${ }^{27,30}$ Soslovsky et al. implemented a murine model $^{35}$ and tested the tendon mobility under several simulated conditions. They provided crucial knowledge about increasing repair tension over time following tendon injury, and the negative impact of high tension on rotator cuff repair in vivo. ${ }^{8,9}$ Unfortunately, despite its anatomic resemblance to the human shoulder, a rat model provides no easy transfer to human in vivo use due to the considerable difference in size and acting forces. Nevertheless, we have found similar characteristics of force-displacement curves compared to in vivo measurements of the murine supraspinatus.

Pastor et al. recently published a study about the impact of reposition technique on tendon tension, using frozen cadaver shoulders. ${ }^{32}$ In limitation, prior studies found altered biomechanical properties for frozen cadaver ${ }^{2}$ and the reported values for tendon tension differed substantially compared to in vivo findings.

Davidson et al. investigated mobility of the human rotator cuff in vivo. ${ }^{4}$ Using a rudimentary device (vicryl tag sutures fixed at the distal tendon stump and attached to a mechanical tensiometer outside the shoulder), they measured the tendon tension needed for rotator cuff repair. They showed a significantly better subjective and objective outcome for those reconstructions with low tendon mobility. Kim et al. showed an inverse correlation of repair tension with healing of reconstructed tendon. ${ }^{16}$ Recently Park et al. found a significant higher re-tear rate when tension $>35 \mathrm{~N}$ is required for reconstruction. ${ }^{31}$

The mobility of the torn tendon in surgical procedures has previously been addressed. Lo and Burkhardt reported favorable results after the arthroscopic interval slide procedure. ${ }^{25}$ Kim et al., however, found higher re-tear rates in patients after an aggressive interval release. ${ }^{18}$ They assumed an increased risk for devascularization with the additional posterior slide. Using the SEAG to verify if an appropriate level of mobilization has achieved might help here. Recently there has been much discussion about single vs. double-row refixation of the rotator cuff tendons. ${ }^{3,11,40} \mathrm{In}$ this regard, a new re-tear type has been described. ${ }^{20}$ The "medial failure", occurs proximal to the reconstruction side at the musculotendinous junction and might be caused by tendon strangulation and stress concentration. Consequently, the attention for a low tension repair is increasing. Double-row refixation has benefits in terms of tendon-bone contact, mechanical strength, and contact pressure. It demonstrates better anatomic healing in many studies. However, a medialized single-row refixation seems favorable for retracted tendons. ${ }^{24}$ Here the influence of tendon strangulation, reduced blood flow, and overtensioning is suspected. Choosing the appropriate surgical technique is difficult, as no absolute values regarding tol- 
erance are available. The SEAG might therefore be beneficial for both scientific investigations and clinical practice.

The in vivo use of the method presented has considerable restrictions. The test station, which enables accurate lateralization, cannot be used in vivo. We assume that only the absolute tension on the tendon at complete reposition is relevant for in vivo use. This can easily be measured using the SEAG.

Human and porcine shoulders are very different. In particular, the porcine supraspinatus seems to have a different tendon-muscle ratio in favor of a pronounced muscle and a shorter tendon. However, published data regarding that observation were not found. In this study, we only created artificial supraspinatus defects by detaching the tendon from its footprint. This is most comparable to a non-retracted acute tendon rupture, which is quite rare in clinical practice. In fact, the majority of patients with rotator cuff tears have a retraction of the tendon, which is mostly due to the degenerative cause of a rotator cuff. Including shoulders with degenerative rotator cuff tears might lead to deviant results. Our measurements of tendon mobility were found to be quite similar to existing data for human in vivo supraspinatus tendons.

As this study aimed to test the reliability of the SEAG, a control group which allows a distinction between degenerative and acute rupture was not tested. ${ }^{16,31}$

It would be very interesting to compare tendon mobility characteristics of healthy and degenerative shoulders in future studies.

We performed the measurements under constant temperature $\left(20^{\circ} \mathrm{C} \pm 0.5\right)$ to minimize the possible influence of rigor mortis. Under these conditions, we found no signs of rigor mortis within a measurement period of $2 \mathrm{~h}$, which is consistent with previously published literature. ${ }^{38}$ Nevertheless, we found no discriminating biomechanical data about the impact of rigor mortis on larger animals, especially for the myotendinous unit of the supraspinatus.

With this feasibility study, we introduce an in vivolike model for investigating rotator cuff tendon mobility. The mobility assessment of the myotendinous unit of fresh porcine shoulder gives a reliable measurement. With the SEAG, we have introduced a reliable device for the future in vivo assessment of tendon mobility in research and clinical settings.

\section{ACKNOWLEDGEMENTS}

Open Access funding provided by Projekt DEAL.

\section{CONFLICT OF INTEREST}

All authors declare that they have no conflict of interest.

\section{OPEN ACCESS}

This article is licensed under a Creative Commons Attribution 4.0 International License, which permits use, sharing, adaptation, distribution and reproduction in any medium or format, as long as you give appropriate credit to the original author(s) and the source, provide a link to the Creative Commons licence, and indicate if changes were made. The images or other third party material in this article are included in the article's Creative Commons licence, unless indicated otherwise in a credit line to the material. If material is not included in the article's Creative Commons licence and your intended use is not permitted by statutory regulation or exceeds the permitted use, you will need to obtain permission directly from the copyright holder. To view a copy of this licence, visit http://crea tivecommons.org/licenses/by/4.0/.

\section{REFERENCES}

${ }^{1}$ Burkhart, S. S. The principle of margin convergence in rotator cuff repair as a means of strain reduction at the tear margin. Ann. Biomed. Eng. 32:166-170, 2004.

${ }^{2}$ Cartner, J. L., Z. M. Hartsell, W. M. Ricci, and P. Tornetta, 3rd. Can we trust ex vivo mechanical testing of fresh-frozen cadaveric specimens? The effect of postfreezing delays. J. Orthop. Trauma 25:459-461, 2011.

${ }^{3}$ Chen, Y., H. Li, Y. Qiao, Y. Ge, Y. Li, Y. Hua, J. Chen, and S. Chen. Double-row rotator cuff repairs lead to more intensive pain during the early postoperative period but have a lower risk of residual pain than single-row repairs. Knee Surg. Sports Traumatol. Arthrosc. 27:3180-3187, 2019.

${ }^{4}$ Davidson, P. A., and D. W. Rivenburgh. Rotator cuff repair tension as a determinant of functional outcome. $J$. Shoulder Elbow Surg. 9:502-506, 2000.

${ }^{5}$ Domb, B. G., R. E. Glousman, A. Brooks, M. Hansen, T. Q. Lee, and N. S. ElAttrache. High-tension double-row footprint repair compared with reduced-tension single-row repair for massive rotator cuff tears. J. Bone Joint Surg. Am. 90(Suppl 4):35-39, 2008.

${ }^{6}$ Edelstein, L., S. J. Thomas, and L. J. Soslowsky. Rotator cuff tears: what have we learned from animal models? $J$. Musculoskelet. Neuronal Interact. 11:150-162, 2011.

${ }^{7}$ Fessel, G., K. Frey, A. Schweizer, M. Calcagni, O. Ullrich, and J. G. Snedeker. Suitability of Thiel embalmed tendons for biomechanical investigation. Ann. Anat. 193:237-241, 2011.

${ }^{8}$ Gimbel, J. A., S. Mehta, J. P. Van Kleunen, G. R. Williams, and L. J. Soslowsky. The tension required at repair to reappose the supraspinatus tendon to bone rapidly increases after injury. Clin Orthop Relat Res 426:258-265, 2004. 
${ }^{9}$ Gimbel, J. A., J. P. Van Kleunen, S. Mehta, S. M. Perry, G. R. Williams, and L. J. Soslowsky. Supraspinatus tendon organizational and mechanical properties in a chronic rotator cuff tear animal model. J. Biomech. 37:739-749, 2004.

${ }^{10}$ Halder, A., M. E. Zobitz, F. Schultz, and K. N. An. Mechanical properties of the posterior rotator cuff. Clin Biomech (Bristol, Avon) 15:456-462, 2000.

${ }^{11}$ Hohmann, E., A. Konig, C. J. Kat, V. Glatt, K. Tetsworth, and N. Keough. Single- versus double-row repair for full-thickness rotator cuff tears using suture anchors. A systematic review and meta-analysis of basic biomechanical studies. Eur. J. Orthop. Surg. Traumatol. 28:859868, 2018.

${ }^{12}$ Itoi, E., L. J. Berglund, J. J. Grabowski, F. M. Schultz, E. S. Growney, B. F. Morrey, and K. N. An. Tensile properties of the supraspinatus tendon. J. Orthop. Res. 13:578584, 1995.

${ }^{13}$ Kawakami, J., N. Yamamoto, H. Nagamoto, and E. Itoi. Minimum distance of suture anchors used for rotator cuff repair without decreasing the pullout strength: a biomechanical study. Arthroscopy 34:377-385, 2018.

${ }^{14}$ Killian, M. L., L. M. Cavinatto, S. R. Ward, N. Havlioglu, S. Thomopoulos, and L. M. Galatz. Chronic degeneration leads to poor healing of repaired massive rotator cuff tears in rats. Am. J. Sports Med. 43:2401-2410, 2015.

${ }^{15}$ Kim, J. H., I. T. Hong, K. J. Ryu, S. T. Bong, Y. S. Lee, and J. H. Kim. Retear rate in the late postoperative period after arthroscopic rotator cuff repair. Am. J. Sports Med. 42:2606-2613, 2014.

${ }^{16}$ Kim, D. H., Y. H. Jang, Y. E. Choi, H. R. Lee, and S. H. Kim. Evaluation of repair tension in arthroscopic rotator cuff repair: does it really matter to the integrity of the rotator cuff? Am. J. Sports Med. 44:2807-2812, 2016.

${ }^{17}$ Kim, Y. K., K. H. Jung, J. W. Kim, U. S. Kim, and D. H. Hwang. Factors affecting rotator cuff integrity after arthroscopic repair for medium-sized or larger cuff tears: a retrospective cohort study. J. Shoulder Elbow Surg. 27:1012-1020, 2018.

${ }^{18}$ Kim, S. J., S. H. Kim, S. K. Lee, J. W. Seo, and Y. M. Chun. Arthroscopic repair of massive contracted rotator cuff tears: aggressive release with anterior and posterior interval slides do not improve cuff healing and integrity. $J$. Bone Joint Surg. Am. 95:1482-1488, 2013.

${ }^{19}$ Kim, Y. K., S. H. Moon, and S. H. Cho. Treatment outcomes of single- versus double-row repair for larger than medium-sized rotator cuff tears: the effect of preoperative remnant tendon length. Am. J. Sports Med. 41:2270-2277, 2013.

${ }^{20}$ Ladermann, A., F. K. Christophe, P. J. Denard, and G. Walch. Supraspinatus rupture at the musclotendinous junction: an uncommonly recognized phenomenon. $J$. Shoulder Elbow Surg. 21:72-76, 2012.

${ }^{21}$ Lake, S. P., K. S. Miller, D. M. Elliott, and L. J. Soslowsky. Effect of fiber distribution and realignment on the nonlinear and inhomogeneous mechanical properties of human supraspinatus tendon under longitudinal tensile loading. J. Orthop. Res. 27:1596-1602, 2009.

${ }^{22}$ Lake, S. P., K. S. Miller, D. M. Elliott, and L. J. Soslowsky. Tensile properties and fiber alignment of human supraspinatus tendon in the transverse direction demonstrate inhomogeneity, nonlinearity, and regional isotropy. J. Biomech. 43:727-732, 2010.
${ }^{23}$ Landis, J. R., and G. G. Koch. The measurement of observer agreement for categorical data. Biometrics 33:159174, 1977.

${ }^{24}$ Lee, K. W., K. H. Moon, C. H. Ma, G. S. Lee, D. S. Yang, and W. S. Choy. Clinical and radiologic outcomes after medializing and not medializing rotator cuff tendon attachment site on chronic retracted rotator cuff tears. Arthroscopy 34:2298-2307, 2018.

${ }^{25}$ Lo, I. K., and S. S. Burkhart. Arthroscopic repair of massive, contracted, immobile rotator cuff tears using single and double interval slides: technique and preliminary results. Arthroscopy 20:22-33, 2004.

${ }^{26}$ Loew, M., P. Magosch, S. Lichtenberg, P. Habermeyer, and F. Porschke. How to discriminate between acute traumatic and chronic degenerative rotator cuff lesions: an analysis of specific criteria on radiography and magnetic resonance imaging. J. Shoulder Elbow Surg. 24:1685-1693, 2015.

${ }^{27}$ Mannava, S., W. F. Wiggins, K. R. Saul, J. D. Stitzel, B. P. Smith, L. A. Koman, T. L. Smith, and C. J. Tuohy. Contributions of neural tone to in vivo passive muscle-tendon unit biomechanical properties in a rat rotator cuff animal model. Ann. Biomed. Eng. 39:1914-1924, 2011.

${ }^{28}$ Meyer, D. C., K. Wieser, M. Farshad, and C. Gerber. Retraction of supraspinatus muscle and tendon as predictors of success of rotator cuff repair. Am. J. Sports Med. 40:2242-2247, 2012.

${ }^{29}$ Mihata, T., T. Fukuhara, B. J. Jun, C. Watanabe, and M. Kinoshita. Effect of shoulder abduction angle on biomechanical properties of the repaired rotator cuff tendons with 3 types of double-row technique. Am. J. Sports Med. 39:551-556, 2011.

${ }^{30}$ Miller, K. S., B. K. Connizzo, and L. J. Soslowsky. Collagen fiber re-alignment in a neonatal developmental mouse supraspinatus tendon model. Ann. Biomed. Eng. 40:11021110, 2012.

${ }^{31}$ Park, S. G., B. J. Shim, and H. G. Seok. How much will high tension adversely affect rotator cuff repair integrity? Arthroscopy 35:2992-3000, 2019.

${ }^{32}$ Pastor, M. F., M. Kraemer, M. Schwarze, C. Hurschler, T. Smith, and M. Wellmann. The effect of rotator cuff malreduction on tendon tension: an evaluation of a custom-made digital tensiometer clamp. Arch. Orthop. Trauma Surg. 138:219-225, 2018.

${ }^{33}$ Patte, D. Classification of rotator cuff lesions. Clin. Orthop. Relat. Res. 254:81-86, 1990.

${ }^{34}$ Shimokobe, H., M. Gotoh, H. Honda, H. Nakamura, Y. Mitsui, T. Kakuma, T. Okawa, and N. Shiba. Risk factors for retear of large/massive rotator cuff tears after arthroscopic surgery: an analysis of tearing patterns. J. Orthop. Surg. Res. 12:140, 2017.

${ }^{35}$ Soslowsky, L. J., J. E. Carpenter, C. M. DeBano, I. Banerji, and M. R. Moalli. Development and use of an animal model for investigations on rotator cuff disease. J. Shoulder Elbow Surg. 5:383-392, 1996.

${ }^{36}$ Tauro, J. C. Arthroscopic, "interval slide" in the repair of large rotator cuff tears. Arthroscopy 15:527-530, 1999.

${ }^{37}$ Thomazeau, H., E. Boukobza, N. Morcet, J. Chaperon, and F. Langlais. Prediction of rotator cuff repair results by magnetic resonance imaging. Clin. Orthop. Relat. Res. 344:275-283, 1997.

${ }^{38}$ Van Ee, C. A., A. L. Chasse, and B. S. Myers. Quantifying skeletal muscle properties in cadaveric test specimens: effects of mechanical loading, postmortem time, and freezer storage. J. Biomech. Eng. 122:9-14, 2000. 
${ }^{39}$ Vollner, F., U. Pilsl, B. Craiovan, F. Zeman, M. Schneider, M. Worner, J. Grifka, and M. Weber. Stability of knee ligament complex of Thiel-embalmed cadaver compared to in vivo knee. J. Mech. Behav. Biomed. Mater. 71:392-396, 2017.

${ }^{40}$ Yang, J., M. Robbins, J. Reilly, T. Maerz, and K. Anderson. The clinical effect of a rotator cuff retear: a meta-analysis of arthroscopic single-row and double-row repairs. Am. J. Sports Med. 45:733-741, 2017.

Publisher's Note Springer Nature remains neutral with regard to jurisdictional claims in published maps and institutional affiliations. 